\title{
Neural Mechanisms of Object Naming and Word Comprehension in Primary Progressive Aphasia
}

\author{
Robert S. Hurley, Ken A. Paller, Emily J. Rogalski, and M. Marsel Mesulam \\ Cognitive Neurology and Alzheimer's Disease Center, Northwestern University, Chicago, Illinois 60611
}

Primary progressive aphasia (PPA) is a neurodegenerative syndrome that causes a gradual atrophy of the left hemisphere language network, leading to impairments of object naming (anomia) and word comprehension. In 33 human subjects with PPA, object naming and word comprehension were explored with $\mathrm{N} 400$ potentials elicited by picture-word or picture-picture matching tasks. Two mechanisms of impairment were identified. In one group of patients, where the object name could be recognized but not retrieved during verbal naming, N400s in picture-word trials were also abnormal, revealing an associative basis for retrieval anomia. In these patients, a putative prephonological signal (i.e., lemma) evoked by the object picture appears to have become too weak to elicit retrieval, but not necessarily too weak to support the informationally less taxing process of recognition. A second group of PPA patients showed more severe naming deficits - the object name was neither verbalized nor recognized. Furthermore, nouns of the same category (but not those of other object categories) could not be identified as mismatches. This blurring of intracategory but not intercategory differentiation of word meaning was correlated with anterior temporal atrophy, predominantly in the left hemisphere, especially along the superior temporal gyrus. Although not part of the classic language network, this area appears critical for proceeding from generic to specific levels of word comprehension and object naming. N400 abnormalities emerged for lexical (picture-word) but not nonverbal (picture-picture) associations, supporting a dual-route rather than amodal organization of object concepts.

\section{Introduction}

The naming of visual objects is an immensely complicated process that requires the perceptual encoding of the object and its rapid linkage to the noun that denotes it (DeLeon et al., 2007; Mesulam et al., 2009a). Object-naming impairments ("anomias") arise if the perceptual process is degraded (as in apperceptive agnosia), if the object does not evoke the multimodal associations related to its nature and use (as in visual associative agnosia), if the noun that denotes the object is not understood (as in Wernicke's aphasia), or if the object representation cannot be linked to the lexical representation of the relevant noun (as in optic aphasia).

The neural substrates of anomia have traditionally been investigated in patients with cerebrovascular accidents (DeLeon et al., 2007). Recently, research on naming and anomia has focused on the neurodegenerative syndrome of primary progressive aphasia

Received Nov. 30, 2011; revised Feb. 9, 2012; accepted Feb. 14, 2012.

Author contributions: R.S.H., K.A.P., and M.M.M. designed research; R.S.H. performed research; R.S.H. and E.J.R. analyzed data; R.S.H., K.A.P., E.J.R., and M.M.M. wrote the paper.

This work was supported by National Institute on Deafness and Communication Disorders Grant DC000552 and National Institute on Aging Grant AG13854 (Alzheimer's Disease Center). Support for R.S.H. was provided by the Training Grant in the Neuroscience of Human Cognition (National Institute of Neurological Disorders and Stroke Grant T32 NS047987) and the Mechanisms of Aging and Dementia Training Grant (National Institute on Aging Grant T32 AG20506). We thank Joseph Boyle and Christina Wieneke for assistance with neuropsychological assessments and EEG application. Tessa Harrison and Adam Martersteck provided assistance with cortical thickness analyses. We also thank Kara Federmeier for comments and feedback on preliminary results.

The authors declare no competing financial interests.

Correspondence should be addressed to Robert S. Hurley at the above address. E-mail: hurley@ northwestern.edu.

DOI:10.1523/JNEUROSCI.5984-11.2012

Copyright $\odot 2012$ the authors $\quad 0270-6474 / 12 / 324848-08 \$ 15.00 / 0$
(PPA). The PPA syndrome provides a unique setting in which components of the left hemisphere temporosylvian language network undergo gradual and selective dissolution. The most recent classification system recommends a subdivision of PPA into agrammatic/nonfluent, logopenic, and semantic variants (Mesulam et al., 2009b; Gorno-Tempini et al., 2011). A major distinction is the impairment of word comprehension in the semantic variant (PPA-S) but not in the agrammatic and logopenic variants (collectively referred to henceforth as "PPA-GL"). The PPA-GL patients display atrophy in classic left perisylvian language regions including the temporoparietal junction and inferior frontal gyrus, whereas PPA-S patients display peak atrophy in the more anterior parts of the temporal lobe (Lambon Ralph et al., 2001; Gorno-Tempini et al., 2004; Mesulam et al., 2009b).

In a previous study on PPA, we used event-related potentials (ERPs) to probe the rapid linkage of visual object percepts to their lexical labels. ERPs were recorded during a task designed so that an object picture prime was followed by a word probe that was either the matching noun that denotes the object, a semantically related mismatch from the same category, or a semantically unrelated mismatch. Our first experiment showed that N400 potentials (Kutas and Hillyard, 1980) were highly sensitive to associative abnormalities underlying anomia in PPA, regardless of clinical subtype (Hurley et al., 2009). These ERP abnormalities were observed despite relatively high accuracy in button press responses, suggesting that predictive coding may be disrupted even in situations in which word recognition remains intact. However, that study did not address the domain specificity of semantic distortions associated with naming failures in PPA. Is anomia caused by a disruption of object representations, lexical 
Table 1. Demographic and language profiles for each group

\begin{tabular}{lccc}
\hline & PPA-S & PPA-GL & Control \\
& $N=9(5 \mathrm{~F})$ & $N=24(11 \mathrm{~F})$ & $N=23(12 \mathrm{~F})$ \\
\hline Age & $62.3(6.5)$ & $66.5(8)$ & $63(6.3)$ \\
Education & $15.4(2.7)$ & $16.2(2)$ & $15.7(2.6)$ \\
WAB-AQ & $80(8)^{*}$ & $78.7(21.5)^{*}$ & $99.7(0.7)^{a}$ \\
BNT & $10.7(10.7)^{*, * *}$ & $39(18.1)^{*}$ & $58.5(2)$ \\
PPVT & $14.9(4.9)^{*, * *}$ & $32.7(3.5)^{*}$ & $35.2(1.4)$ \\
PPT-Pictures & $41.2(5.4)^{*, * *}$ & $50.1(1.3)^{*}$ & $51.2(1.1)$ \\
PPT-Words & $39.3(6.6)^{* * * *}$ & $49.8(2.1)^{*}$ & $51.7(7)$ \\
WAB-Rep & $88.2(7.6)^{*}$ & $71.2(27.2)^{*}$ & $99.1(1.9)$ \\
NAT & $8.1(2.8)^{*}$ & $7.1(2.3)^{*}$ & $9.7(0.7)$ \\
WPM & $90(17.9)^{*}$ & $93.6(40.5)^{*}$ & $131.9(19.6)^{a}$ \\
\hline
\end{tabular}

Values represent mean (SD). All groups were matched for age and years of education. PPA patients were sorted into two groups, PPA-S and PPA-GL. Both PPA groups showed lower performance on all language measures compared with controls. PPA-S patients showed lower performance than PPA-GL patients on measures of naming and semantic judgment. Education, Years of education; WAB-AQ, -Rep, Western Aphasia Battery-Aphasia Quotient, -Repetition; BNT, Boston Naming Test; PPVT, Peabody Picture Vocabulary Test; PPT, Pyramids and Palm Trees Test; NAT, Northwestern Anagram Test; WPM, words per minute.

${ }^{a}$ Based on a separate set of 12 controls who were equivalent in age and years of education to the current control group.

${ }^{*} p<0.05$ versus controls; ${ }^{* *} p<0.05$ versus PPA-GL.

representations, or of their linkage? Is the principal deficit amodal, and therefore verbal as well as nonverbal, or is it selectively greater for lexical representations of objects? These questions were addressed in the current set of experiments by adding a new type of trial in which both prime and probe were object pictures so that the integrity of picture-picture associations could be measured separately from the integrity of picture-word associations (see Fig. 1). Results were also analyzed to test the hypothesis that anomia in PPA-S patients is caused by a distortion in word comprehension and lexical labeling at the specific rather than generic level of identification (Mesulam et al., 2009a).

\section{Materials and Methods}

\section{Participants}

Twenty-three control participants (12 females) and 33 PPA patients (16 females) were recruited at the Northwestern University Cognitive Neurology and Alzheimer's Disease Center. Diagnosis of PPA required a history of language impairments unaccompanied by consequential decline in other cognitive domains for at least the first 2 years of the disease (Mesulam, 2003). To meet research criteria for the presence of aphasia, patients were required to have abnormal performance on the Western Aphasia Battery (WAB) (Kertesz, 1982). Patients were also assigned clinical subtypes (Mesulam et al., 2009b; Gorno-Tempini et al., 2011). Single-word comprehension was assessed by a 36-item subset of the Peabody Picture Vocabulary Test (PPVT) (Dunn and Dunn, 2007), in which participants are given an array of pictures and asked to select an item after hearing its name aloud. Nine patients were assigned a PPA-S subtype based on scores $<60 \%$ on the PPVT (Mesulam et al., 2009b). Grammar deficits were assessed by scores on the Northwestern Anagrams Test (NAT) (Weintraub et al., 2009), in which patients are given words on cards and are asked to arrange the cards to form syntactically correct sentences. Of the patients with intact PPVT performance, 10 were assigned a PPA-G subtype based on performance below $60 \%$ on the NAT, and the remaining 14 patients were assigned a PPA-L subtype as comprehension and grammar were relatively intact. Data from the PPA-G and PPA-L patients were collapsed into a single PPA-GL group $(n=24)$, as both groups showed similar results in preliminary analyses, and investigations of object-naming function were focused on the influence of word comprehension deficits. The two patient groups, PPA-GL and PPA-S, were distinguished by the presence of word comprehension impairments in the latter but not the former. The control, PPA-S, and PPA-GL groups were of similar demographic composition (Table 1), with equivalent ages $\left(F_{(2,53)}=1.8 ; p=0.18\right)$, years of education $\left(F_{(2,53)}=\right.$ $0.39 ; p=0.68)$, and proportions of each gender $(p=0.88$, Fisher's exact test). Both PPA groups showed lower performance on all language mea- sures compared with controls, with PPA-S patients showing even lower performance than PPA-GL patients on measures of naming, as assessed by the Boston Naming Test (Kaplan et al., 1983) and semantic judgment as assessed by the Pyramids and Palm Trees Test (Howard et al., 1992). Two of the PPA-GL patients were left-handed and were excluded from MRI analyses.

\section{Naming and comprehension of experimental stimuli}

Stimuli were 48 concrete nouns and corresponding black-and-white photograph depictions of each object from a mixture of living and nonliving categories (i.e., animals, tools, fruits, etc.). To maximize difficulty in naming and stimuli judgments, these items were of lower than average frequency [average log CELEX frequency of 0.31 (SD, 0.33)] (Baayen et al., 1995).

To gather further information on the processing of pictures and words used in the ERP experiment, PPA subjects were also asked to perform the following three supplementary tests with the experimental stimuli before the recording session.

Picture naming. Patients were asked to name aloud each of the 48 picture stimuli. This was a timed procedure, and participants were required to name aloud each item within $6 \mathrm{~s}$ of picture onset. This information was later used to classify the stimuli into nameable versus unnameable items. Phonemic paraphasic distortions were considered as correctly named for purposes of this analysis, while all other paraphasic errors, "don't know," and absent responses were classified as unnamed.

Word comprehension assessed by word-to-picture pointing. Comprehension of the nouns denoting each object was assessed by word-to-picture pointing, in which patients were given the target noun and asked to point to the relevant object from among an array of all 48 objects.

Categorical sorting. Knowledge of category structure was then assessed by asking patients to sort cards with pictures or written nouns depicting each item. Patients were asked to place each word and picture card into one of five bins-animals, vegetables, kitchenware, office supplies, outside tools- each bin labeled with the written name of the category and a cluster of exemplar pictures. Controls were not asked to complete these supplementary tests.

\section{Matching task paradigm}

During ERP testing, stimuli appeared as prime-probe pairs in one of three delivery platforms: picture-picture, picture-word, and word-word (Fig. 1), and participants were asked to judge whether each pair matched or not. There was a total of 576 trials total (192 per platform), with trials from each platform intermixed rather than blocked throughout the experiment. The experiment lasted $50 \mathrm{~min}$ on average, with breaks provided every 10 min. Even PPA-S patients with severe word comprehension deficits showed high accuracy on word-word trials, raising the possibility that patients were judging the visual similarity between written word primes on a perceptual level rather than accessing the deeper semantic meaning of those words. Given this ambiguity in interpretation, data from the word-word condition were excluded from subsequent analyses. Congruity between primes and probes was manipulated such that they matched on one-half of trials and mismatched on one-half. On picture-word trials, matching probes consisted of the corresponding noun denoting the object picture (96 trials). On picture-picture trials, one-half of matching probes were the exact same picture presented twice in a row ( 48 trials), and one-half were a different example photograph of the same object (e.g., two different types of toasters; 48 trials). For the sake of clarity, data are presented only from the latter type of "exemplar" picture-picture match (Fig. 1), as this condition is more conceptually demanding and therefore more appropriate for comparison with the matching picture-word conditions. Probes on related mismatch trials (48 trials for each platform) were pictures or written words from another item in that same category (e.g., pear and pineapple). Unrelated probes were words and pictures from a different category (e.g., tiger and glue; also 48 trials per platform). Stimuli were counterbalanced such that each item appeared as a matching, related, and unrelated probe in both the word and picture platforms. Primes and probes were presented on each trial for $800 \mathrm{~ms}$ each, with a $200 \mathrm{~ms}$ interstimulus interval (Fig. 1). Participants were asked to press one button on matching trials, and another 


$\begin{array}{cc}\begin{array}{c}\text { Picture Prime } \\ \text { (800ms) }\end{array} & \text { Interval } \\ \text { (200ms) }\end{array}$

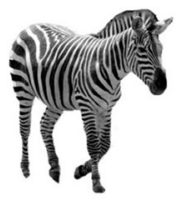

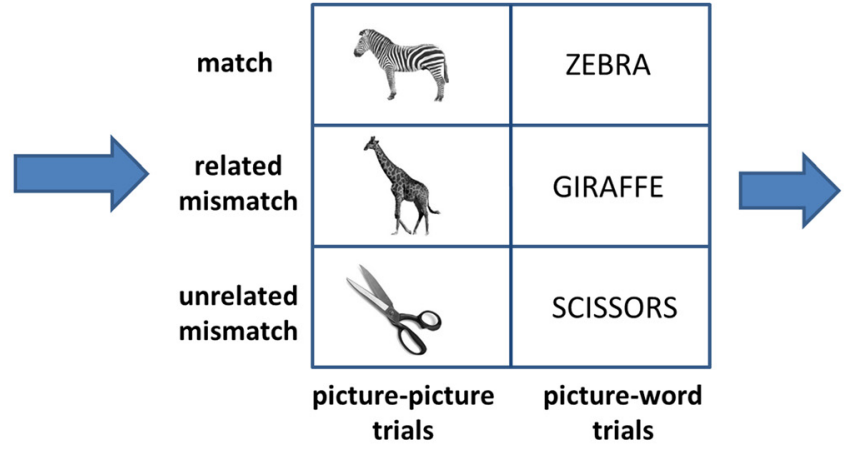

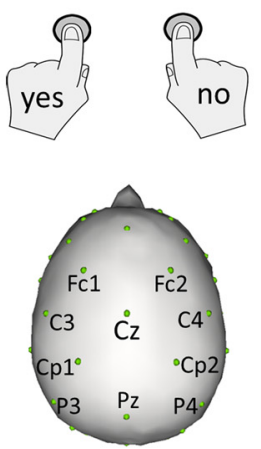

Is the Probe a Match?

EEG recorded from

scalp electrodes

Figure 1. Schematic of the ERP matching task. The task is based on a prime followed by a probe. The N400 was triggered by the probe and reflects the semantic discrepancy between the associations evoked by the prime and the identity of the probe. Primes in all trials were photographs of concrete objects presented for $800 \mathrm{~ms}$. After a $200 \mathrm{~ms}$ interval (blank screen), the probe (object picture or written word) was presented for $800 \mathrm{~ms}$. For picture-picture trials, the probe was another object photograph, and for picture-word trials, the probe was a written word. Matching primes were the written name of the object or (for picture-picture trials) another example picture of the same object. Mismatches were of two types: related mismatches came from the same category as the prime, and unrelated mismatches came from a different category. Participants responded by pressing one button for matches and another for mismatches. EEG was recorded from 32 scalp electrodes. Amplitude values from the 10 dorsal electrodes shown were used in inferential analyses of N400 effects.

button for mismatched trials (both related and unrelated). The interval between trials randomly varied between 2.5 and $3.5 \mathrm{~s}$.

\section{Analysis of ERP data}

EEG was recorded from 32 scalp electrodes in an elastic cap, using a BioSemi ActiveTwo high-impedance amplifier (BioSemi Instrumentation). EEG signals were acquired at a sampling rate of $512 \mathrm{~Hz}$, and rereferenced off-line to averaged mastoid channels. EEG data were bandpass filtered from 0.1 to $100 \mathrm{~Hz}$, with an additional $59-61 \mathrm{~Hz}$ notch filter to reduce AC background noise. Data were epoched from -100 to $800 \mathrm{~ms}$ relative to word onset, and baseline corrected to the $100 \mathrm{~ms}$ prestimulus interval. Electro-ocular artifacts were monitored using electrodes placed below and lateral to the eyes. An eyeblink correction algorithm was implemented using EMSE software (Source Signal Imaging) to remove blinks from the EEG trace. Epochs with remaining electro-ocular and other muscle artifacts were excluded from analysis. This resulted in an average (SD) of 41.8 (5.5) trials per condition contributing to ERPs in controls, 37.8 (7.4) trials in PPA-GL patients, and 42.2 (5.8) trials in PPA-S. Inferential analyses were based on average ERP amplitude from 350 to $550 \mathrm{~ms}$ after probe onset, across 10 dorsal electrode locations (Fig. 1). Significance of the N400 mismatch effect was evaluated by repeatedmeasures ANOVA contrasts between matching and mismatched conditions (i.e., contrast weights of $-2 / 1 / 1$ for match/related/unrelated), collapsed across 10 electrode sites.

\section{Analysis of MRI data}

Structural MR images were available on 28 of 33 PPA patients, including all 9 of the PPA-S patients. MR images were collected using a T1weighted 3D MPRAGE sequence (repetition time, $2300 \mathrm{~ms}$; echo time, $2.86 \mathrm{~ms}$; flip angle, $9^{\circ}$; field of view, $256 \mathrm{~mm}$ ) recording 160 slices at a thickness of $1.0 \mathrm{~mm}$ acquired with a Siemens Trio 3 tesla scanner, using a 12-channel birdcage head coil. Imaging was performed at the Northwestern University Department of Radiology Center for Advanced MRI. Cortical thickness analyses were conducted using the FreeSurfer image analysis suite (http://surfer.nmr.mgh.harvard.edu/). After warping into a common space, cortical thickness was calculated as the distance between the gray matter/white matter boundary and the pial surface (Fischl and Dale, 2000), resulting in thickness estimates with submillimeter spatial resolution. Statistical surface maps were generated using a general linear model (GLM) that displayed differences in cortical thickness between the PPA and healthy groups for each vertex along surface representations of the entire neocortex. In addition to the whole-brain analysis, which identified the patterns of significant atrophy, statistical GLM surface maps were computed to identify significant relationships

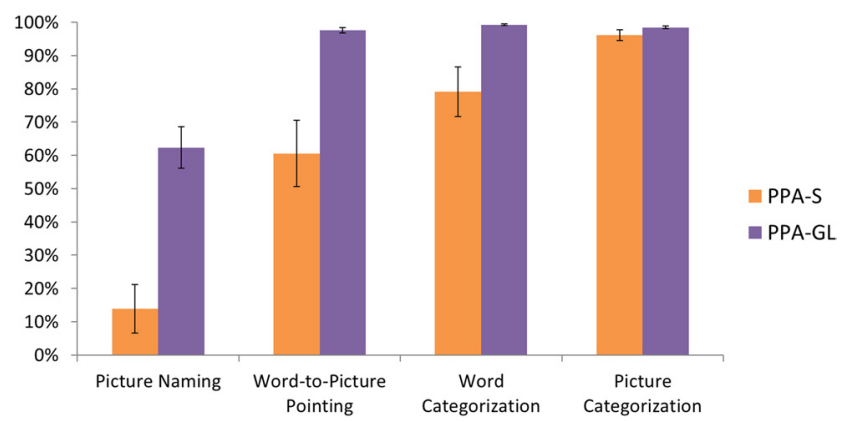

Figure 2. Naming, recognition, and categorization of objects used as primes. Several behavioral tasks were administered before the ERP recording session. In Picture Naming, patients were asked to name aloud each of the 48 experimental picture stimuli used as primes. In Word-to-Picture Pointing, patients were shown the written name of an object, one at a time, and asked to point to the corresponding picture in an array containing pictures of all 48 objects. In Word Categorization and Picture Categorization, they were then given 96 cards, each containing one of the 48 pictures or the corresponding 48 nouns, to sort into generic semantic categories (e.g., animals, tools, etc.). PPA-S patients showed pronounced impairments in Picture Naming and Word-to-Picture Pointing, suggesting that the anomia is based, at least in part, on the inability to recognize (or understand) the noun that denotes the specific object. The better performance in Word Categorization is likely to reflect a relative sparing of word comprehension at the more general categorical level than at the specific level required by the Word-to-Picture Pointing task. Error bars indicate SEM.

between cortical thickness in each cerebral hemisphere and ERP amplitudes.

\section{Results}

\section{Pre-ERP testing}

Naming and comprehension of the experimental word and picture stimuli were assessed before ERP testing (see Materials and Methods). PPA-GL patients were able to name aloud $62 \%$ of the 48 pictures used in the ERP testing, while PPA-S patients were able to name only $13 \%$ of picture stimuli (Fig. 2). In word-topicture pointing, which is sensitive to word comprehension, PPA-S patients again showed low accuracy (61\%), while PPA-GL patients showed near-ceiling performance (98\%). Category sorting was more successful in PPA-S patients who correctly sorted $79 \%$ of words and $96 \%$ of pictures. Sorting is a relatively easier task 
A

Control

(n:23)

PPA-GL

(n:24)

PPA-S

(n:9)

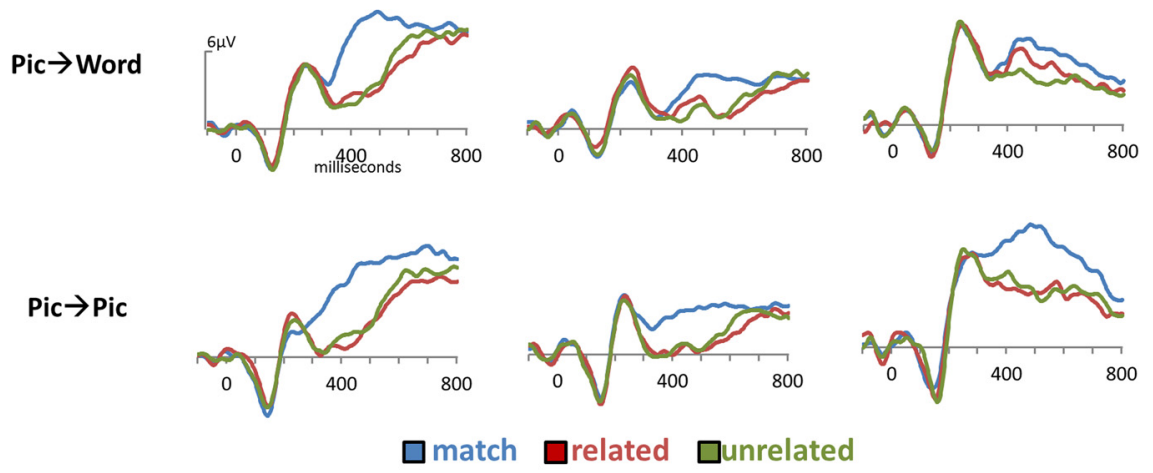

B Picture $\rightarrow$ Word

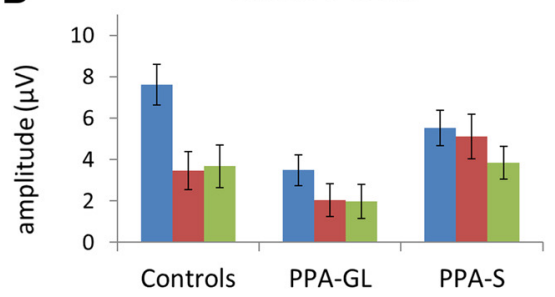

C

Picture $\rightarrow$ Word

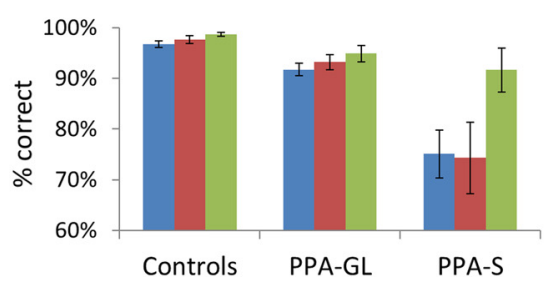

Picture $\rightarrow$ Picture
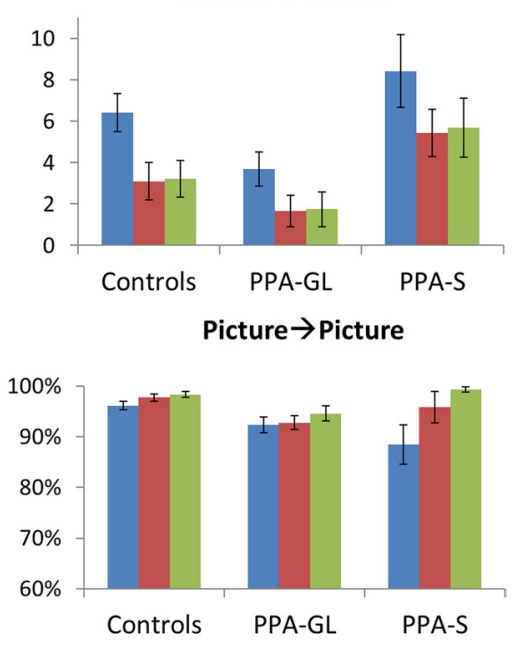

Figure 3. ERP matching task results. $A$, ERPs in response to the three types of probes are shown from the vertex electrode (Cz). Controls showed more negative ERPs from 350 to 550 ms (i.e., N400) in response to both related and unrelated word and picture probes compared with matching probes, comprising an N400 mismatch effect. The N400 mismatch effect was significant in PPA-GL patients, but of smaller magnitude compared with controls. PPA-S patients showed an N400 mismatch effect for unrelated but not related word probes, suggesting a blurring of neural responses to nouns that denote objects within the same object category. In contrast, ERPs to picture probes were of equivalent amplitude in all three groups. $\boldsymbol{B}, \mathrm{N} 400$ amplitudes corresponding to the waveforms in $A$ are extracted from 350 to $550 \mathrm{~ms}$ after probe onset, averaged across the 10 dorsal electrodes used for interferential analyses. C, Controls and PPA-GL patients were highly accurate at the matching task. PPA-S patients showed distinctly worse performance on trials with matching as well as related word probes, showing further evidence of word recognition impairment and blurred distinctions between nouns that denote objects of the same category (i.e., the match and related mismatch). Error bars indicate SEM.

that could conceivably be influenced by common perceptual features of objects. Nevertheless, the differential performance of PPA-S patients in word-to-picture pointing versus word categorization could be indicating that word comprehension is more impaired at the specific level of identification required by the former than at the level of more general comprehension that is sufficient for the latter. Performance in category sorting was nearly perfect in PPA-GL (>98\%).

\section{ERPs in response to picture and word probes}

N400 potentials were evident during a 350-550 ms interval following probe presentation. Mean amplitude measurements within this interval revealed a robust N400 "mismatch effect" in controls (Fig. 3A,B); ERPs were significantly more negative in response to related as well as unrelated mismatches, compared with the match. This mismatch effect was significant in controls during both picture-word trials $\left(F_{(1,22)}=77.7 ; p<0.001\right)$ and picture-picture trials $\left(F_{(1,22)}=32.1 ; p<0.001\right)$.

As a group, PPA-GL patients also showed a significant N400 mismatch effect in picture-word trials $\left(F_{(1,23)}=15.6\right.$; $p=0.001)$ and in picture-picture trials $\left(F_{(1,23)}=24.9 ; p<0.001\right)$, although the magnitude of this match-mismatch amplitude differential was reduced compared with controls selectively in pictureword trials $\left(F_{(1,45)}=18.7 ; p<0.001\right)$. In PPA-S, the N400 mismatch effect was not significant in picture-word trials in which the mismatch was related $\left(F_{(1,8)}=0.64\right.$; $p=0.45)$, while responses to unrelated mismatches remained significant $\left(F_{(1,8)}=7.88\right.$; $p=0.023)$ and of equivalent amplitude to controls $\left(F_{(1,30)}=0.149 ; p=0.703\right)$. This dissociation shows that neural responses in PPA-S can differentiate words that denote different object categories but not words that denote different objects within the same category. The N400 mismatch effect in PPA-S was significant in picture-picture trials for both related and unrelated mismatches $\left(F_{(1,8)}=10.4 ; p=0.012\right)$ with an amplitude equivalent to that in controls $\left(F_{(1,30)}=0.144 ; p=0.71\right)$.

The major neurophysiological abnormalities were that (1) N400 mismatch effects in PPA-GL were decreased for related and unrelated mismatch words and that (2) N400 mismatch effects in PPA-S were absent for categorically related mismatch words. N400 mismatch effects were generally normal in both groups for both types of mismatch pictures. Because PPA-S patients named only $13 \%$ of the pictures, the N400 patterns in Figure 3 largely reflect responses to unnamed items. This impression was confirmed by an additional analysis in which only trials with unnamed items $(87 \%$ of total) were included; N400 mismatch effects were present for unrelated words but absent for related words $\left(F_{(1,8)}=7.33, p=\right.$ 0.027, and $F_{(1,8)}=0.21, p=0.66$, respectively).

Push button responses in determining whether the probe is a match or mismatch

Controls and PPA-GL patients showed high accuracy ( $\geq 92 \%)$ during the push button responses of the ERP task (Fig. 3C). PPA-S patients showed particularly low accuracy in response to the match $(75 \%)$ and related mismatch $(74 \%)$ in the pictureword trials (vs unrelated trials, $F_{(1,8)}=40.328, p<0.001$ ). The low accuracy in response to the match indicates difficulty in recognizing (i.e., understanding) that the word is the name of the object, while the additional inaccuracy in response to the related (but not unrelated) mismatch indicates a selective difficulty in differentiating match from related mismatch, words that belong to the same object category.

Reaction times were calculated on trials with accurate responses. All groups were slower to respond to related compared with unrelated mismatches (controls, $F_{(1,22)}=82.6, p<0.001$; PPA-GL, $F_{(1,23)}=36.9, p<0.001$; PPA-S, $F_{(1,8)}=16.9, p=$ 
A

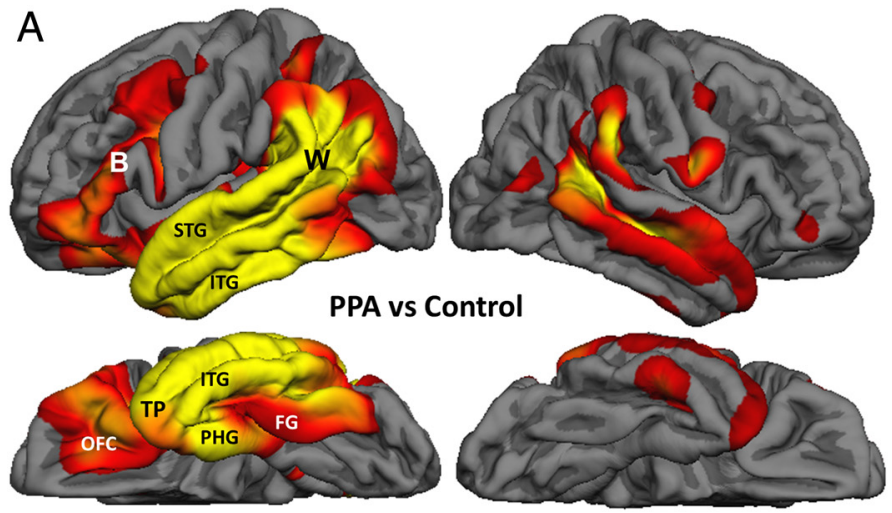

C

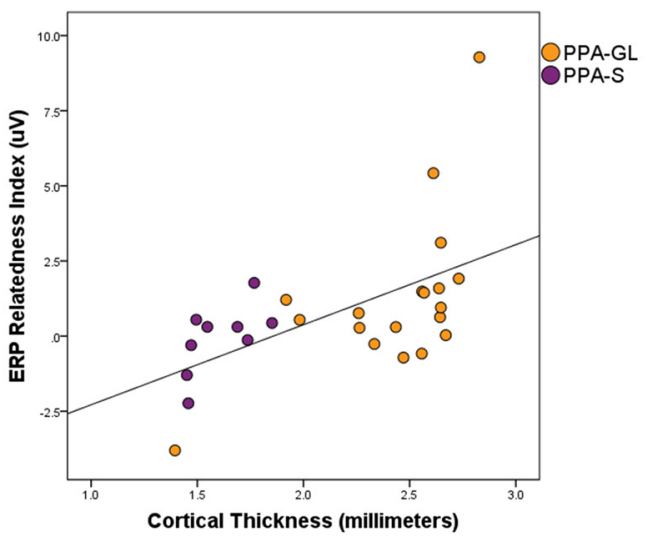

B

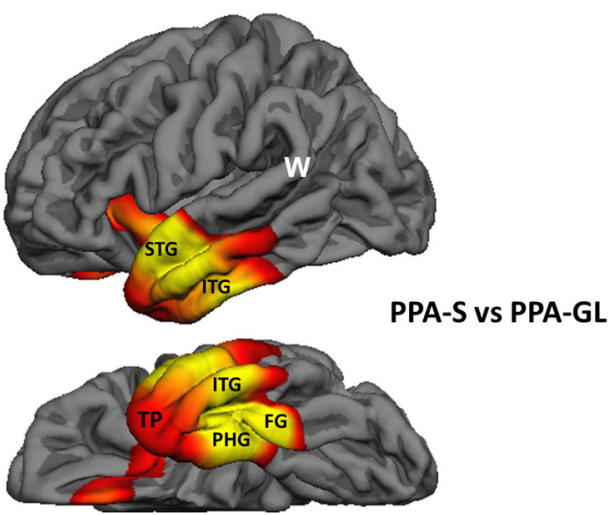

Correlations of atrophy with relatedness index

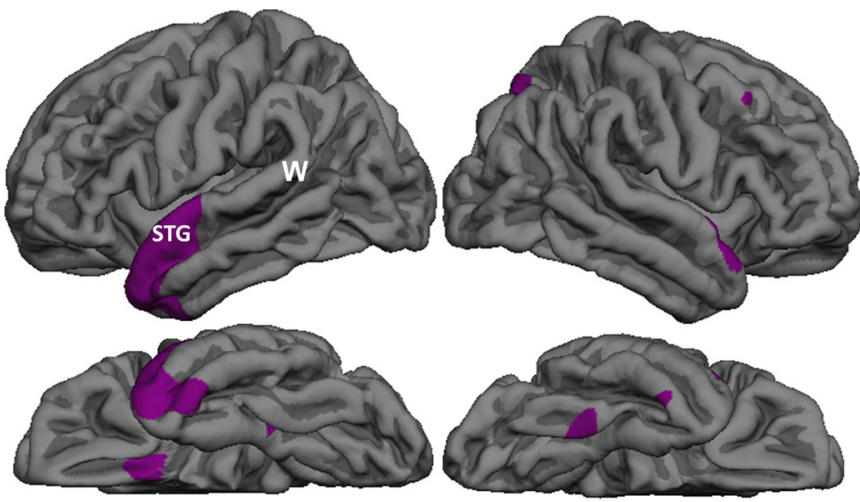

Figure 4. Relationships between cortical thickness and ERPs. Structural MRI scans were available on 28 of the 33 PPA patients. $A$, The PPA group as a whole (19 PPA-GL and 9 PPA-S) showed peak atrophy in the temporoparietal junction (Wernicke's area), the inferior frontal gyrus (Broca's area), lateral and inferior temporal cortex, and the orbitofrontal area (A). Significance of thinning is displayed as $\log _{10}(p)$ values in yellow/red, thresholded by an FDR of $p<0.001$ in each hemisphere [ $\mathrm{min} / \mathrm{max} \log _{10}(p)$ values $=3.4 / 5.7$, left hemisphere; 3.9/6.2, right hemisphere]. $\boldsymbol{B}$, Compared with the PPA-GL group, the PPA-S group showed more severe atrophy throughout the anterior temporal lobe ( $\left.\min / \max \log _{10}(p)=4.0 / 6.2\right)$. C, A relatedness index was calculated for picture-word trials by dividing the differential amplitude to a related mismatch (compared with a match) by the differential amplitude to an unrelated mismatch. With the exception of one PPA-GL patient, the PPA-S patients had the lower values of this index, reflecting their selective difficulty in differentiating words of the same object category. $\boldsymbol{D}$, To clarify which part of the atrophy in $\boldsymbol{A}$ was most closely associated with the decline of the relatedness index, a correlation analysis was performed. The results showed significant correlations $(p<0.01)$ in the anterior part of the STG, and the TP. Both areas are more severely atrophied in PPA-S, which also has the lowest relatedness index and greatest difficulty in differentiating words of the same category. Calso shows the quantitative basis of the correlation between decreased relatedness index and atrophy in the left anterior temporal area shaded in $\mathbf{D}$. Abbreviations: B, Broca's area; $\mathrm{FG}$, fusiform gyrus; ITG, inferior temporal gyrus; $0 \mathrm{FC}$, orbitofrontal cortex; PHG, perihippocampal gyrus; STG, superior temporal gyrus; TP, temporal pole; W, Wernicke's area.

0.003), revealing a "semantic interference" effect (La Heij, 1988; Starreveld and La Heij, 1995). PPA-S patients were also slower to respond to matching words $\left(t_{(8)}=2.7 ; p=0.026\right)$ than to unrelated mismatches, but not to matching pictures $\left(t_{(8)}=1.5 ; p=\right.$ 0.16 ), another reflection of the difficulty in recognizing (i.e., understanding) the match as the name of the object. In contrast, controls and PPA-GL patients showed equivalent reaction times on matching and unrelated picture-word trials, indicating that they recognized the match as the name of the pictured object (controls, $t_{(22)}=0.38, p=0.71$; PPA-GL, $t_{(23)}=0.72, p=0.48$ ).

\section{Anatomical correlates of the intracategory blurring}

A rigorous false-discovery rate (Genovese et al., 2002) of $p<$ 0.001 was applied to the results from each FreeSurfer GLM, to reveal areas of peak atrophy in each hemisphere. The PPA group as a whole (19 PPA-GL and 9 PPA-S patients) showed peak atrophy in the temporoparietal junction (Wernicke's area), the inferior frontal gyrus (Broca's area), lateral and inferior temporal cortex, and the orbitofrontal area of the left hemisphere (Fig. $4 A$ ), compared with a previously collected sample of 27 controls (14 males; 13 females) of similar age and education (Rogalski et al., 2011). Atrophy patterns were similarly distributed but distinctly less extensive in the right hemisphere. Compared with the PPA-GL group, the PPA-S group showed more severe atrophy throughout the left anterior temporal lobe (Fig. 4B). A "relatedness index" was calculated for picture-word trials by dividing the change in N400 amplitude to related mismatches (matchrelated) by the change in amplitude to unrelated mismatches (match-unrelated). This index reflects the ability of the N400 to distinguish two words of the same object category as normalized by the ability to distinguish two words of different categories. With the exception of one PPA-GL patient, the PPA-S patients had the lower values of this index, reflecting their selective difficulty in differentiating words of the same object category (Fig. $4 C$ ). To clarify which part of the atrophy in Figure $4, A$ and $B$, was most closely associated with the decline of the relatedness index, a correlation analysis was performed. The results showed significant correlations $(p<0.01)$ predominantly in the anterior part of the left superior temporal gyrus (STG), and the temporal pole (TP) (Fig. 4D). Both areas are more severely atrophied in PPA-S group, which also has the lowest relatedness index and greatest difficulty in differentiating words of the same category. Addi- 


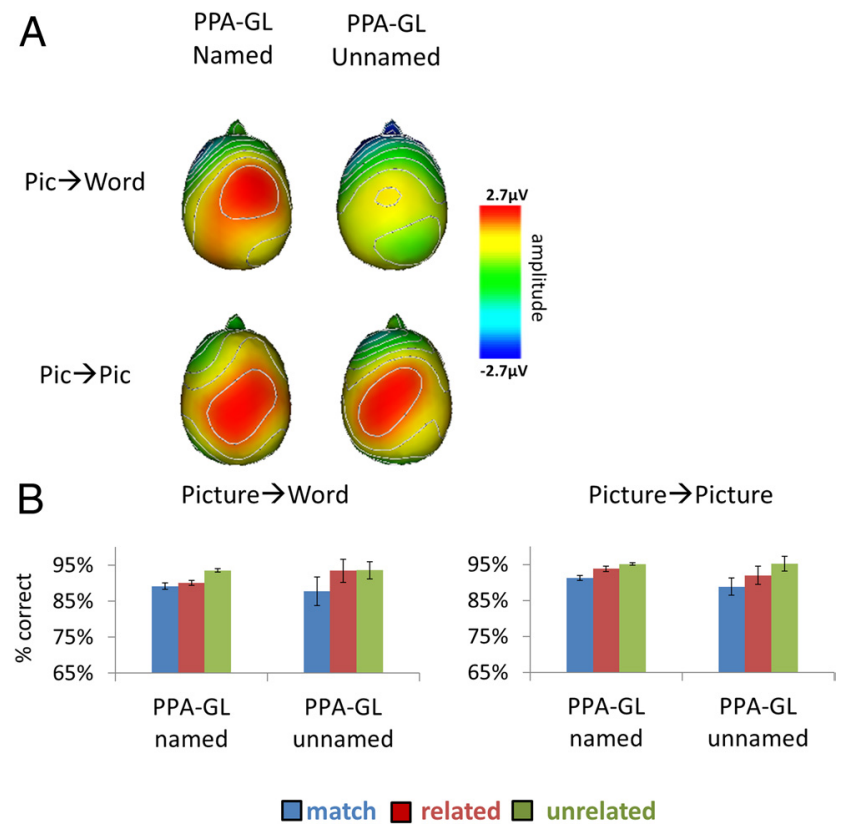

Figure 5. Responses to named and unnamed items in PPA-GL. $\boldsymbol{A}$, Topographic plots of the N400 mismatch effect (responses to matched vs related and unrelated mismatched probes) from nine anomic PPA-GL patients. Red denotes a robust $\mathrm{N} 400$ effect, whereas yellow and green indicate low amplitude or absent responses. The plots show the widespread and selective absence of $\mathrm{N} 400$ to related and unrelated mismatches specifically for unnamed objects but only in the picture-word trials. $\boldsymbol{B}$, Accuracy of performance during the ERP matching task according to naming success. PPA-GL patients show similar accuracy on both named and unnamed word and picture trials. The ERP abnormalities implicate associative failures in lexical access (or retrieval) even though the patients recognized (i.e., understood) the word that denoted the pictures they could not name. Error bars indicate SEM.

tional significant correlations of lesser extent were seen at the anterior STG and small patch of fusiform gyrus in the right hemisphere. Figure $4 C$ provides quantitative documentation of the correlation between atrophy and loss of the relatedness index in the entire group of patients included in the MRI analysis, averaged across the left anterior temporal region shaded in Figure $4 D$.

\section{The anomia of PPA-GL}

The 24 PPA-GL patients displayed a wide range of naming performance in the pre-ERP testing (Fig. 2). We identified a subset of nine PPA-GL patients who had comparable numbers of named (59\%) and unnamed (41\%) items, so their ERPs could be examined separately by naming success (Fig. $5 A$ ). In trials in which the object prime had been named aloud in pre-ERP testing, the N400 mismatch effect (for both related and unrelated mismatches) trended toward significance on picture-picture trials $\left(F_{(1,8)}=\right.$ $5.0 ; p=0.056)$, and was significant on picture-word trials $\left(F_{(1,8)}=\right.$ $6.3 ; p=0.036)$ but of lower magnitude compared with controls $\left(F_{(1,30)}=7.2 ; p=0.011\right)$. Although the quantitative analysis of the N400 for named picture-picture trials in PPA-GL fell just shy of significance, the topographic maps (Fig. $5 \mathrm{~A}$ ) show that its magnitude and distribution are essentially intact. In contrast, on trials in which the prime object could not be named, the PPA-GL group showed a loss of the N400 mismatch effect in picture-word $\left(F_{(1,8)}=0.93 ; p=0.36\right)$ but not picture-picture trials $\left(F_{(1,8)}=\right.$ $7.3 ; p=0.027$ ) (Fig. $5 A$ ). In other words, there was a loss of the N400 effect on picture-word trials only for items that could not be named. The loss of N400 effects occurred even though the behavioral accuracy for recognizing matches and mismatches was close to $90 \%$ (Fig. $5 B$ ), showing that the patients correctly recognized (i.e., understood) the word they could not retrieve during overt naming and that there was no differential impairment in recognizing the related versus unrelated mismatch, of the type seen in PPA-S.

\section{Discussion}

We used ERP methodology in an experiment in which an object picture (prime) was followed by picture or word probes. There were three probe types: a "match" of the prime, a "related mismatch" representing another object of the same category, or an "unrelated mismatch" representing an object of a different category. The task was to press one button for a match and another for a mismatch. The N400, also known as the incongruity potential, is normally larger in response to mismatching probes. The N400 elicited by related mismatches indicates sensitivity to intracategory distinctions, whereas the $\mathrm{N} 400$ in response to unrelated mismatches indicates sensitivity to intercategory distinctions. The picture-picture trials assess the integrity of this process within the object recognition network, whereas the picture-word trials assess the additional ability to interlink the language and object networks in a paradigm that simulates object naming. In picture-word trials, push button accuracy in response to the matching word indicates the recognition (i.e., comprehension) of the word as the correct name of the object. Moreover, the differential N400 and push button performance in response to related versus unrelated mismatches reflect the capacity for word comprehension and object naming at the generic versus more fine-grained and specific levels of encoding. These assumptions guided the interpretation of the experimental results.

We identified two distinctive types of anomia, one based on impaired retrieval (i.e., lexical access) and the other on distorted semantic representation of words (i.e., lexical semantics). In PPA-GL patients, objects that could not be named aloud by confrontation elicited accurate push button responses and word-topicture pointing. This pattern indicates that the name of the object could be recognized and linked to the correct object but could not be retrieved, raising the possibility of a postsemantic bottleneck in accessing phonological representations of the noun. If that were the case, the N400 should have been intact, as was shown in one patient who could write the names of objects she was unable to verbalize during confrontation naming (Hurley et al., 2009). In the PPA-GL patients, however, primes depicting objects that could not be named also failed to elicit mismatch N400 potentials (Fig. $5 A$ ), showing that the anomia results from an associative failure (Fig. $5 B$ ). The problem cannot be attributed to a distortion of nonverbal object representations, since the N400s in the picture-picture trials were intact, but rather appears to reflect weakness of a putative prephonological signal (i.e., lemma) evoked by the object picture. Naming failure in PPA-GL arises when this signal is too weak to elicit retrieval, but not necessarily too weak to support the informationally less taxing process of recognition. The retrieval (or access) anomia in PPA thus has an associative component that leads to a distortion, but not obliteration, of the interface between nonverbal object representations and their lexical labels.

A different anomia mechanism was identified in PPA-S patients. In the picture-word trials for objects that could not be named, the N400 was lost selectively in response to the related mismatch. This pattern indicates that the remaining neurons in these patients were less efficient in differentiating words from the same category than words from different categories (Fig. $3 A, B$ ). This dissociation did not reflect a problem in retrieval, as in the case of PPA-GL, since push button accuracy was low in response to the match, indicating difficulty in recognizing the word as the 
name of the object. The patients were also more accurate in recognizing the categorically unrelated word as a mismatch than the categorically related one (Fig. 3 C), providing behavioral evidence for a greater preservation of intercategory than intracategory distinctions in object labeling and word comprehension. Further support for this conclusion comes from the pre-ERP behavioral tasks in which the PPA-S patients were less able to demonstrate word comprehension at the specific level required by the wordto-picture pointing than in the word categorization task that could be solved at a more generic level. It appears therefore that the anomia in PPA-S is based on a mechanism that interferes with the progression of word concepts from the generic to specific level of precision and their linkage to objects. The abnormality cannot be attributed to a distortion of nonverbal object representations since these patients, despite the severe anomia, continue to show normal N400s and push button performance in the picture-picture trials.

To explore the anatomical correlates of this distortion in semantic mapping, we computed a "relatedness index" in the picture-word trials, defined as the change in N400 amplitude between matching and related words, divided by the difference in amplitude between matching and unrelated words. This ratio reflected the ability to form distinct lexical associations within a given category, normalized by the differential responses to words from other categories. As expected, this index was lower in the PPA-S patient group than in the PPA-GL group (Fig. 4C). The relatedness index was then correlated with cortical atrophy to determine the location of neuronal loss most closely associated with the blurring of intracategory distinctions. Intracategory semantic blurring was specifically seen in PPA-S patients, who also had more severe atrophy broadly throughout the left temporal lobe including the fusiform and parahippocampal gyri (Fig. 4B). Nonetheless, significant correlations with the relatedness index were confined to a predominantly left-sided anterior subset of these areas that included STG, TP, and an adjacent patch of inferior temporal gyrus (ITG) (Fig. $4 D$ ). Neuronal loss in these predominantly left-sided anterior temporal regions appears to mediate the intracategory blurring of lexical representations and the resultant distortions in the specificity of word comprehension and object naming. Our approach was designed to detect such correlates within areas of atrophy in the PPA group. We therefore cannot rule out the possibility that there are other regions that mediate similar functions in the intact brain.

The correlation analysis suggests that selective damage to anterior STG is likely to distort the more fine-grained differentiation of words, compared with more posterior STG lesions within Wernicke's area, which would presumably lead to more generalized comprehension failures, at least according to accounts of classic aphasiology (DeLeon et al., 2007) (but see Walker et al., 2011, for conflicting views). Left anterior temporal lesions have been linked with selective loss in naming unique concrete entities such as famous faces and buildings (Damasio et al., 2004; Tranel, 2006), providing further evidence that this region mediates finegrained semantic differentiation. This localization is also consistent with investigations of cerebrovascular lesions that revealed a hereto neglected role of anterior temporal cortex in the finegrained mapping of concepts to words (Schwartz et al., 2009; Walker et al., 2011), and functional MRI experiments that show anterotemporal activations during word comprehension tasks (Gitelman et al., 2005). This evidence thus suggests that the classic Wernicke-Lichtheim-Geschwind language network is incomplete and that the left anterior temporal lobe, especially the anterior STG and adjacent temporal pole, should be inserted as a third major hub of the language network with a special role in the hierarchical processing of word meaning and object naming from general to more specific levels of precision.

Anterotemporal cortex has recently attracted a great deal of attention as a critical site for semantic processing (Simmons and Martin, 2009). Influential accounts, derived predominantly from investigations of semantic PPA variants, have proposed the presence of an "amodal" representation of objects in the temporal pole (McClelland and Rogers, 2003; Patterson et al., 2007). Such a view would predict that objects with impaired lexical associations should also have impaired nonverbal associations (Lambon Ralph and Patterson, 2008). Our results do not support such an organization. In PPA-S patients, we found a selective distortion in verbal but not nonverbal associations triggered by the picture of an object. This dissociation is consistent with a "dual-route" model according to which the left perisylvian language network is distinct from a bilateral inferotemporal object representation network (Mesulam, 1998). Accordingly, severe disruptions of object concepts can be confined to their verbal or nonverbal associations, in keeping with cognitive theories specifying separate stores for visual and verbal semantic knowledge (Paivio, 1986). Object naming requires an interaction of the two networks. Bilateral inferotemporal lesions would be expected to trigger anomias caused principally by distortions of object concepts, whereas left hemisphere language network lesions should lead to anomias caused principally by distortions of lexical concepts. The distinctions are never absolute since damaging either network interferes with the function of the other through the mechanism of disconnection. In our PPA-S sample, anomia was more closely linked to a distortion of lexical than nonverbal associations because our patients were chosen to have relatively isolated language impairments as required by PPA diagnostic criteria (Mesulam, 2003; Gorno-Tempini et al., 2011). We therefore infer that the object recognition network was relatively spared. In previous investigations of semantic dementia, in which the same diagnostic criteria for PPA may not have been used, patients with more bilateral lesions are likely to have been included, leading to observations in which impairment of verbal associations become difficult to separate from impairments of nonverbal associations.

Primary progressive aphasia is a unique disease of the language network. In contrast to cerebrovascular accidents, PPA leads to a slow and partial loss of constituent neurons. The resultant perturbations of language function can thus be more subtle and informative than those seen in cerebrovascular lesions. The current study, based on the high temporal resolution of eventrelated potentials, shows that PPA can provide unique insights into the neural organization of lexical access and comprehension.

\section{References}

Baayen RH, Piepenbrock R, Gulikers L (1995) The CELEX lexical database (CD-ROM). Philadelphia: Linguistic Data Consortium, University of Pennsylvania.

Damasio H, Tranel D, Grabowski T, Adolphs R, Damasio A (2004) Neural systems behind word and concept retrieval. Cognition 92:179-229.

DeLeon J, Gottesman RF, Kleinman JT, Newhart M, Davis C, Heidler-Gary J, Lee A, Hillis AE (2007) Neural regions essential for distinct cognitive processes underlying picture naming. Brain 130:1408-1422.

Dunn LM, Dunn DM (2007) Peabody Picture Vocabulary Test PPVT-4. San Antonio, TX: Pearson Assessments.

Fischl B, Dale AM (2000) Measuring the thickness of the human cerebral cortex from magnetic resonance images. Proc Natl Acad Sci U S A 97:11050-11055.

Genovese CR, Lazar NA, Nichols T (2002) Thresholding of statistical maps in functional neuroimaging using the false discovery rate. Neuroimage 15:870-878. 
Gitelman DR, Nobre AC, Sonty S, Parrish TB, Mesulam MM (2005) Language network specializations: an analysis with parallel task designs and functional magnetic resonance imaging. Neuroimage 26:975-985.

Gorno-Tempini ML, Dronkers NF, Rankin KP, Ogar JM, Phengrasamy L, Rosen HJ, Johnson JK, Weiner MW, Miller BL (2004) Cognition and anatomy in three variants of primary progressive aphasia. Ann Neurol 55:335-346.

Gorno-Tempini ML, Hillis AE, Weintraub S, Kertesz A, Mendez M, Cappa SF, Ogar JM, Rohrer JD, Black S, Boeve BF, Manes F, Dronkers NF, Vandenberghe R, Rascovsky K, Patterson K, Miller BL, Knopman DS, Hodges JR, Mesulam MM, Grossman M (2011) Classification of primary progressive aphasia and its variants. Neurology 76:1006-1014.

Howard D, Patterson KE; Thames Valley Test Company. (1992) The Pyramids and Palm Trees Test. Suffolk, UK: Thames Valley Test Company.

Hurley RS, Paller KA, Wieneke CA, Weintraub S, Thompson CK, Federmeier $\mathrm{KD}$, Mesulam MM (2009) Electrophysiology of object naming in primary progressive aphasia. J Neurosci 29:15762-15769.

Kaplan E, Goodglass H, Weintraub S (1983) Boston Naming Test. Philadelphia: Lea and Febiger.

Kertesz A (1982) The Western Aphasia Battery. New York; London: Grune and Stratton.

Kutas M, Hillyard SA (1980) Reading senseless sentences: brain potentials reflect semantic incongruity. Science 207:203-205.

La Heij W (1988) Components of Stroop-like interference in picture naming. Mem Cognit 16:400-410.

Lambon Ralph MA, Patterson K (2008) Generalization and differentiation in semantic memory: insights from semantic dementia. Ann N Y Acad Sci 1124:61-76.

Lambon Ralph MA, McClelland JL, Patterson K, Galton CJ, Hodges JR (2001) No right to speak? The relationship between object naming and semantic impairment: neuropsychological evidence and a computational model. J Cogn Neurosci 13:341-356.

McClelland JL, Rogers TT (2003) The parallel distributed processing approach to semantic cognition. Nat Rev Neurosci 4:310-322.

Mesulam MM (1998) From sensation to cognition. Brain 121:1013-1052.
Mesulam MM (2003) Primary progressive aphasia—a language-based dementia. N Engl J Med 349:1535-1542.

Mesulam M, Rogalski E, Wieneke C, Cobia D, Rademaker A, Thompson C, Weintraub S (2009a) Neurology of anomia in the semantic variant of primary progressive aphasia. Brain 132:2553-2565.

Mesulam M, Wieneke C, Rogalski E, Cobia D, Thompson C, Weintraub S (2009b) Quantitative template for subtyping primary progressive aphasia. Arch Neurol 66:1545-1551.

Paivio A (1986) Mental representations: a dual coding approach. New York; Oxford: Oxford UP; Clarendon.

Patterson K, Nestor PJ, Rogers TT (2007) Where do you know what you know? The representation of semantic knowledge in the human brain. Nat Rev Neurosci 8:976-987.

Rogalski E, Cobia D, Harrison TM, Wieneke C, Thompson CK, Weintraub S, Mesulam MM (2011) Anatomy of language impairments in primary progressive aphasia. J Neurosci 31:3344-3350.

Schwartz MF, Kimberg DY, Walker GM, Faseyitan O, Brecher A, Dell GS, Coslett HB (2009) Anterior temporal involvement in semantic word retrieval: voxel-based lesion-symptom mapping evidence from aphasia. Brain 132:3411-3427.

Simmons WK, Martin A (2009) The anterior temporal lobes and the functional architecture of semantic memory. J Int Neuropsychol Soc 15:645-649.

Starreveld PA, La Heij W (1995) Semantic interference, orthographic facilitation, and their interaction in naming tasks. J Exp Psychol Learn Mem Cogn 21:686-698.

Tranel D (2006) Impaired naming of unique landmarks is associated with left temporal polar damage. Neuropsychology 20:1-10.

Walker GM, Schwartz MF, Kimberg DY, Faseyitan O, Brecher A, Dell GS, Coslett HB (2011) Support for anterior temporal involvement in semantic error production in aphasia: new evidence from VLSM. Brain Lang 117:110-122.

Weintraub S, Mesulam MM, Wieneke C, Rademaker A, Rogalski EJ, Thompson CK (2009) The northwestern anagram test: measuring sentence production in primary progressive aphasia. Am J Alzheimers Dis Other Demen 24:408-416. 